\title{
Dynamic Imaging of Hydrate Specific Area Evolution during Xenon Hydrate Formation
}

\author{
Zaher Jarrar \\ zjarrar@vols.utk.edu \\ Civil and Environmental Engineering, University of Tennessee, Knoxville, TN 37996, USA \\ Riyadh Al-Raoush \\ riyadh@qu.edu.qa \\ Department of Civil and Architectural Engineering, Qatar University, Doha, Qatar \\ Khalid Alshibli \\ alshibli@utk.edu \\ Civil and Environmental Engineering, University of Tennessee, Knoxville, TN 37996, USA \\ Jongwon Jung \\ jjung@chungbuk.ac.kr \\ School of Civil Engineering, Chungbuk National University, Cheongju, Chungbuk, South Korea
}

\begin{abstract}
Gas hydrates are ice-like structures formed under high pressure and low temperature conditions. They are considered as a potential energy source due to their abundance and the increase in energy demand worldwide. A fundamental understanding of hydrate formation and dissociation kinetics is essential in order to improve gas productivity from natural hydrates reservoirs. This paper investigates the evolution of hydrate specific area during the process of hydrate formation using dynamic 3D synchrotron microcomputed tomography. Xenon hydrate was formed inside a high-pressure low-temperature cell, filled with silica sand partially saturated with water. The cell has a height of $70.2 \mathrm{~mm}$ and an inner diameter of $9.7 \mathrm{~mm}$, and is capable of sustaining an internal pressure of 150 $\mathrm{MPa}$. During hydrate formation and dissociation, full 3D images are acquired at a time resolution of 45 seconds and a spatial resolution of $5.38 \mu \mathrm{m} /$ voxel. The reconstructed images were enhanced and segmented, and direct volume and surface area measurements were obtained. Initially, the specific area of hydrate increased with increasing hydrate saturation up to a certain hydrate saturation threshold ( $9 \%$ hydrate saturation). After this threshold, hydrate specific area started to decrease with increasing hydrate saturation. This is an indication that the small crystals of hydrates tend to merge and form larger crystals during the process of hydrate formation.
\end{abstract}

Keywords: Gas hydrates; Synchrotron micro-computed tomography; Dynamic imaging; Hydrate formation

\section{INTRODUCTION}

Gas hydrates are ice-like structures formed under high pressure and low temperature conditions where gas (guest molecules) is trapped within a crystal structure of water (host molecules). They are considered as a potential energy source due to their abundance and the increase in energy demand worldwide (Sloan \& Koh, 2008). A fundamental understanding of hydrate formation and dissociation kinetics is essential in order to improve gas productivity from natural hydrates reservoirs. This paper investigates the 
evolution of hydrate specific area during the process of hydrate formation using dynamic 3D synchrotron microcomputed tomography. Xenon hydrate was formed inside a highpressure low-temperature cell, filled with silica sand partially saturated with water. The cell has a height of $70.2 \mathrm{~mm}$ and an inner diameter of $9.7 \mathrm{~mm}$, and is capable of sustaining an internal pressure of $150 \mathrm{MPa}$. During hydrate formation, full 3D images were acquired at a time resolution of 45 seconds and a spatial resolution of $5.38 \mu \mathrm{m} /$ voxel, and direct measurements of hydrate volume and surface area were obtained from the segmented images.

\section{METHODOLOGY}

In this study, pink beam synchrotron microcomputed tomography (PSMT) was used to obtain high-resolution 3D images during hydrate formation. In pink beam tomography, a glazed mirror and an x-ray absorbing foil are used to obtain an x-ray beam with 1000 times higher flux than a monochromatic beam (Rivers, 2016). PSMT images were acquired at beamline 13D, Advanced Photon Source (APS), Argonne National Laboratory (ANL), Illinois, USA.

Xenon hydrate was formed inside a high-pressure low-temperature cell, filled with silica sand partially saturated with water. The cell has a height of $70.2 \mathrm{~mm}$ and an inner diameter of $9.7 \mathrm{~mm}$, and is capable of sustaining an internal pressure of $150 \mathrm{MPa}$. During hydrate formation, full 3D images were acquired at a time resolution of 45 seconds and a spatial resolution of $5.38 \mu \mathrm{m} / \mathrm{voxel}$. The reconstructed images were enhanced and segmented using Avizo commercial software, similar to the procedure described in Jarrar et al. (2018). A sample xy slice of one of the images is depicted in Figure 1, with hydrates shown as bright voxels.

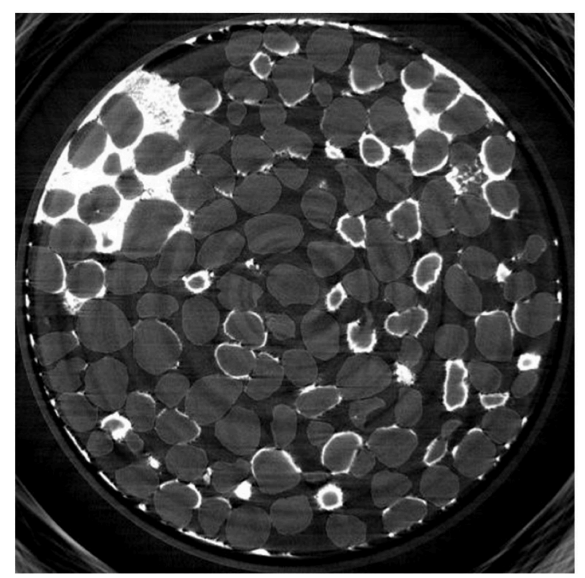

Figure 1: Sample xy slice of one of the images during hydrate formation with hydrates shown as bright voxels.

\section{RESULTS}

The segmented images were used to directly measure the change of hydrate volume and surface area during hydrate formation. Figure 2 shows the change of hydrate specific area (area/volume) with hydrate saturation during Xenon hydrate formation. Each point in Figure 2 represents measurements obtained from a full segmented 3D image. Initially, 
the specific area of hydrate increased with increasing hydrate saturation up to a certain hydrate saturation threshold (which corresponds to about $9 \%$ hydrate saturation). After this threshold, hydrate specific area began to decrease as hydrate saturation increases. This is an indication that the small crystals of hydrates tend to merge, forming larger crystals during the process of hydrate formation.

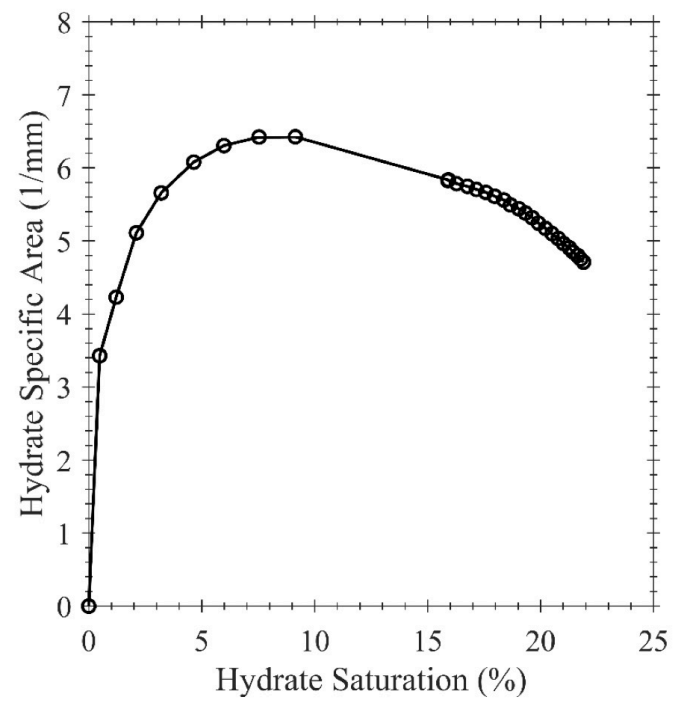

Figure 2: Change of hydrate specific area with hydrate saturation during Xenon hydrate formation.

\section{CONCLUSION}

Xenon hydrate was formed inside a high-pressure low-temperature cell, filled with silica sand partially saturated with water. The cell has a height of $70.2 \mathrm{~mm}$ and an inner diameter of $9.7 \mathrm{~mm}$, and is capable of sustaining an internal pressure of $150 \mathrm{MPa}$. During hydrate formation, full 3D images were acquired at a time resolution of 45 seconds and a spatial resolution of $5.38 \mu \mathrm{m} / \mathrm{voxel}$, and direct measurements of hydrate volume and surface area were obtained from the segmented images. The specific area of hydrate initially increased with increasing hydrate saturation up to a hydrate saturation threshold of $9 \%$, after which, hydrate specific area began to decrease as hydrate saturation increased. This indicates that the small crystals of hydrates tend to merge, forming larger crystals during the process of hydrate formation.

\section{ACKNOWLEDGMENTS}

This publication was made possible by partial funding from NPRP grant \# NPRP8594-2-244 from the Qatar national research fund (a member of Qatar Foundation) and the Institute for a Secure and Sustainable Environment (ISSE), University of TennesseeKnoxville, USA. Any opinions, findings, and conclusions or recommendations expressed in this material are those of the authors and do not necessarily reflect the views of funding agencies. This paper used resources of the Advanced Photon Source (APS), a U.S. Department of Energy (DOE) Office of Science User Facility operated for the DOE Office of Science by Argonne National Laboratory (ANL) under Contract 
No. DE-AC02-06CH11357. The PSMT images presented in this paper were collected using the x-ray Operations and Research Beamline Station 13-BMD at Argonne Photon Source (APS), ANL. We thank Dr. Mark Rivers of APS for help in performing the SMT scans. We also acknowledge the support of GeoSoilEnviroCARS (Sector 13), which is supported by the National Science Foundation, Earth Sciences (EAR-1128799), and the DOE, Geosciences (DE-FG02-94ER14466).

\section{REFERENCES}

Jarrar, Z. A., Al-Raoush, R. I., Hannun, J. A., Alshibli, K. A. \& Jung, J. (2018). 3D synchrotron computed tomography study on the influence of fines on gas driven fractures in Sandy Sediments. Geomechanics for Energy and the Environment, 100-105.

Rivers, M. L. (2016). High-speed tomography using pink beam at GeoSoilEnviroCARS, Developments in X-Ray Tomography X, Stock. Muller, R., Wang, B. \& SPIE, G.

Sloan, E. D. \& Koh, C. A. (2008). Clathrate hydrates of natural gases. CRC Press, Boca Raton, FL, the USA. 